\title{
Écho du passé, reflet du présent : l'Histoire dans deux récits d'Alexandre Dumas
}

\author{
Gaétan-Philippe Beaulière \\ Université d'Ottawa
}

En raison de sa nature intrinsèquement antithétique - que fonde l'alliage de deux discours en apparence irréconciliables, l'Histoire et le récit - , le roman historique problématise de manière aiguë le rapport du texte au réel; dès lors qu'il fait de l'Histoire l'objet d'une représentation, le texte ne peut plus être considéré comme un médiateur neutre ou «fidèle». Claudie Bernard remarque avec justesse dans Le Passé recomposé que " [s]upposer le face-à-face d'un référent stable et d'un récit ("représentant") qui le dénoterait sans autre déformation qu'"artistique" est un mécanisme de défense destiné à 
scotomiser la toute-puissance instable de l'idéologie, qui imprègne la représentation comme acte et le représenté comme sens » (p. 76-77). À l'inverse, n'y voir qu'un pur effet d'idéologie, que la promotion larvée ou inconsciente, mais toujours résolue, d'un projet politique, c'est surévaluer la scientificité d'un discours qui, selon Michel de Certeau, tenant à la fois de la «pratique interprétative » du passé et de la «praxis sociale », n'est aucunement imperméable à un investissement idéologique (p. 29).

Ces questions ne sauraient manquer d'intéresser le lecteur d'Alexandre Dumas; chez l'auteur des Mousquetaires, le passage de l'Histoire à la fiction allait de pair avec un important travail d'interprétation. Ce phénomène est certainement lié à ses velléités pédagogiques qui, couplées au succès fulgurant qu'ont connu ses récits - qui accordaient une importance variable à l'Histoire - l'ont placé au premier rang des romanciers vulgarisateurs du XIX siècle français, rôle qu'il reconnaissait et revendiquait par ailleurs (voir Mombert). Le travail d'interprétation, inhérent à cette entreprise de vulgarisation, est particulièrement clair dans les récits qui forment La Femme au collier de velours et Les Mille et Un Fantômes, où Dumas semble se livrer à une dure et amère critique de la Révolution de 1789 en mettant l'accent sur les excès qu'organisait et promouvait la Terreur. Mais si un lecteur attentif ne peut passer outre la critique inhérente à la représentation de la Terreur dans ces récits, il semble excessif de voir là confirmée la thèse, défendue par Anne-Marie CalletBianco, voulant que l'auteur soit un «nostalgique de la monarchie » (p. 35). 
Il n'en demeure pas moins que la représentation de l'Histoire dans ces deux récits est spéculaire: le travail d'interprétation des faits historiques est, sinon conditionné, à tout le moins orienté par les paradigmes de la période qui les produit. Autrement dit, en filigrane derrière la peinture dumasienne du Paris de 1789, il y a la France d'après la Révolution de 1848. «La différence des perspectives historiques », écrit Georges Lukacs dans Le Roman historique, «conditionne aussi une différence dans les principes de composition et de caractérisation. » (p. 13) ${ }^{1}$ Or, Les Mille et Un Fantômes et La Femme au collier de velours ont été publiés quelque temps après la Révolution de 1848, à laquelle Dumas a pris une part active avant d'échouer à se faire élire député. Le bilan de sa déconfiture électorale le pousse à douter de la valeur d'un suffrage universel (proclamé le 2 mars de la même année), à questionner ce pouvoir remis entre les mains d'un peuple peu instruit, donc dépourvu des connaissances historiques et politiques nécessaires à l'exercice éclairé du droit de vote: il est hautement désabusé quant à la capacité du peuple de faire des choix politiques justes. C'est d'ailleurs ce sentiment de méfiance, au demeurant mâtiné d'un certain populisme propre à l'humanisme bourgeois de l'époque ${ }^{2}$, qui l'avait poussé à fonder $L e M{ }^{3} S^{3}$ le $1^{\text {er }}$ mars 1848 et qui lui

\footnotetext{
1 Nous nous fondons donc sur les postulats de la " théorie marxiste du genre » telle qu'elle est étayée et appliquée par Georges Lukacs, sans toutefois adopter la perspective de la dialectique marxiste (p. 13).

2 Henri Troyat décrit Dumas comme un « républicain modéré, ami de la justice et ennemi du désordre ». Aussi « ce programme nuancé » serait-il selon lui à la base de son engagement politique (p. 380).

3 Ce journal, qui visait à retracer l'ensemble de l'Histoire de France depuis février 1848, avait une visée d'éducation populaire. Une note trouvée sur la page frontispice du premier numéro, stipulait : «M. Alexandre Dumas, ayant
} 
inspira une critique des institutions démocratiques. Qui plus est, désillusionné face à la capacité du peuple de jouer son rôle au sein de l'Histoire, il sera amer à la suite de l'étouffement des révoltes populaires de juin 1848, opéré manu militari par le gouvernement républicain. Les raisonnements politiques étayés dans les Fantômes - et synthétisés dans la lettre à Véron qui les précèdent (p. 241-245) - reflètent ce profond désenchantement. Si l'on fait le pari d'étudier l'interprétation de l'Histoire par Dumas sans chercher à y voir une prise de position pour ou contre la Révolution française, mais plutôt une réflexion critique inspirée de la réalité sociopolitique qui lui est contemporaine, on pourra certainement parvenir à réconcilier les idées véhiculées à travers ces récits avec la pensée politique de l'auteur.

\section{Le peuple : les dérives d'un nouvel acteur sociopolitique}

Dans les deux textes en présence est dénoncée une application erronée et abusive de la notion d'égalité, qui justifie une remise du pouvoir entre les mains de gens dont les compétences sont insuffisantes à l'exercice de leurs fonctions. L'ignorance des fonctionnaires - dont les signatures «ne ressemblaient pas mal à des zigzags d'ivrognes »- causent une lourdeur administrative ayant pour conséquence que «[j]amais la paperasserie n'eut autant de développement qu'à cette époque » (p. 130). Le narrateur va jusqu'à affirmer que, chez les sans-culottes, la lucidité et l'aptitude sont inversement proportionnelles au nationalisme : « Là où le patriotisme était le

voulu faire un journal a porté de tous, à mis le prix de ce journal à QUATRE FRANCS par an » (Dumas, février 1848). 
plus chaud, les écrivains étaient plus naïfs » (p. 130). En raison de leur incapacité à jauger la valeur des institutions et de leur propension à user de la violence, ils sont dépeints comme étant singulièrement dénués de civilité et de goût, notamment dans le VIII e chapitre de La Femme au collier de velours, dont le titre synthétise l'essence du propos du narrateur à cet égard: «Comment les musées et les bibliothèques étaient fermés, mais comment la place de la Révolution était ouverte » (p. 139). Avec cynisme, Dumas se moque d'une dénaturation des idéaux républicains cautionnant les actes prosaïques : « [u]n voyageur était donc, dès son arrivée, fouillé, interrogé, dénudé, quant au moral, avec une facilité que donnait l'usage, et une liberté que donnait... la liberté » (p. 132). Selon le narrateur, les inscriptions cacographiques produites par un maire forment un «parfait document» (p.130), nécessaire à l'accession au «bonheur de savourer cette précieuse forme de gouvernement» (p.129). Le fantastique est également mis au service du propos politique; le récit du chevalier Lenoir, où un ouvrier paie chèrement sa profanation de la dépouille d'Henri IV (il est, dans un premier temps, désavoué par ses amis, puis par la suite poussé à la contrition par les spectres, p. 343-346), se passe de commentaires.

L'opposition entre les situations qu'engendre la Terreur et les idéaux dont elle se réclame offre, dans La Femme au collier de velours, un contraste frappant, que la narration accentue. Les inégalités que la Révolution aurait dû chercher à éradiquer existent toujours; de fait, les pauvres ne peuvent avoir une part de l'argent ravi à l'aristocratie: «[a]insi se heurtait partout ce grand principe d'égalité proclamé par la hache, écrit avec le sang, et sur lequel avaient le droit de cracher en riant les prostituées du Palais-Royal» (p. 203). Il 
n'est pas fortuit que, dans le récit, la maîtresse d'une figure de proue de la Révolution française, Danton, vive dans la surabondance et ne puisse être conquise "qu'à prix d'argent » (p. 197). Le narrateur voit du reste en l'appropriation des biens de la monarchie par le peuple une «dernière ironie contre le passé » (p. 203) : " [l] luxe était abandonné aux filles les plus viles» (p. 203). Les injustices qui avaient motivé le soulèvement du peuple sont reproduites de manière éhontée, tant et si bien que, paradoxalement, les bénéficiaires du nouveau système inique, à cause de leur mépris du passé, portent entrave au progrès. Le Paris de 1793 se révèle, dans $L a$ Femme au collier de velours, un terreau fertile au développement d' « obscénités », du « vice », de la « corruption » (p. 204), du jeu, etc. : «À voir ces gobelets pleins d'or et ces pyramides d'argent, on eût eu bien de la peine à croire que la misère publique était si grande, et que l'or coûtait si cher. » (p. 205) Paris devient dès lors un lieu de perdition. Hoffmann, qui s'y était rendu afin d'étudier la musique, de jouir de ses nombreuses institutions culturelles, y sera plutôt corrompu au point de rompre son serment et causer de facto la mort de sa fiancée et de son beau-père (p. 237).

Il est clair qu'Alexandre Dumas présente la Révolution comme un mouvement dont les idéaux ont été détournés au point de le rendre à bien des égards réactionnaire. Au nom de l'égalité, des ignorants sont investis de pouvoir et des injustices, que cautionne une notion vaste de la liberté, ont certes été perpétuées sous une forme différente, mais toujours au détriment du peuple, dupe de cette «philosophie de l'antithèse » (p. 203). On perçoit, derrière une telle représentation de la dérive collective d'un peuple ignorant, le Dumas éducateur populaire pour qui le manque d'instruction 
rend inepte à jouer un rôle politique d'envergure. Nous ne pourrions pas, en outre, manquer de voir là une attestation de la relation complexe de la bourgeoisie aux classes populaires, relation "oscillant entre vision terrorisée de la plèbe et un populisme superficiel» (Bernard, p.10), qui aurait valu une répudiation de l'électorat au «soi-disant champion des classes laborieuses qui n'a jamais tenu le manche d'un marteau ni conduit une charrue» (Troyat, p. 382) qu'était l'auteur des Mousquetaires.

Lukacs note que c'est le XIXe siècle qui a vu entrer le peuple dans l'Histoire. Plus précisément, de la prise de la Bastille à la Restauration, se sont enchaînées autant d' « expérience[s] vécue[s] des masses »; la prise de conscience par le peuple de son poids socio-historique est ainsi fortement liée aux soubresauts politiques qui ont secoué le XIX siècle (Lukacs, p.21-22), ce qui a naturellement préoccupé les intellectuels de tout acabit : dès lors que le peuple avait été un catalyseur de changement, on a cherché à expliquer son rôle, son statut, son pouvoir. C'est pourquoi il sera tour à tour perçu comme une «multitude sans laquelle on ne peut plus compter», un "réservoir de besoins et d'énergies», un «moteur de l'émancipation sociale» et une "communauté vitale, source de tout héroïsme et de toute vertu », selon qu'on se réclame de l'humanisme, du libéralisme ou de la pensée de Marx. Un éventuel abandon du peuple à ses mécanismes primitifs est craint, puisque, aux yeux de la démocratie, ceux-ci représentent une véritable épée de Damoclès (Bernard, p. 23). Le portrait inquiet de l'action du peuple en 1789 brossé par Alexandre Dumas en est le reflet probant; on peut y lire la préoccupation pressante d'une élite (la bourgeoisie) forcée de 
composer avec le pouvoir grandissant d'une classe jusqu'alors privée d'un accès aux leviers politiques.

\section{La peine de mort ou le paroxysme des outrances populaires}

La peine de mort et le rapport qu'y entretiennent les instigateurs de la Terreur jouent ici un rôle central à la caractérisation du peuple. Que les sans-culottes aient fait de la guillotine une « machine de gouvernement » (Arasse, p. 102) est effectivement révélateur des postulats de leur pensée politique, que les textes en présence cherchent à réfuter. Arasse écrit : «La guillotine est révolutionnaire ou, plus précisément, elle est démocratique au sens où le Comité de salut public entend le gouvernement du peuple, car le fonctionnement de la machine illustre avec éclat une théorie précise de la "démocratie populaire" » (p. 104). Anne-Marie Callet-Bianco n'a pas tort de souligner que, en sa qualité d'immigrant idéalisant Paris, Hoffmann joue un rôle-clé dans cette représentation abjecte de la Terreur, sa désillusion brutale (p. 28) contribuant à la mise en relief du contraste abordé ci-dessus. Par contre, la véritable astuce de l'auteur est d'être parvenu à dénoncer la peine de mort par la bouche de personnages français, républicains de surcroît, mais dégoûtés par les excès de la Terreur. Le discours tenu par l'hôtesse du poète allemand, par exemple, illustre en quoi le partage des idéaux révolutionnaires ne s'accompagne pas nécessairement d'une «canonisation de la guillotine révolutionnaire ${ }^{4}$. Bien qu'elle ne soit aucunement gênée par le

\footnotetext{
4 Daniel Arasse rapporte que la guillotine « devient l'objet d'un culte dont les formules et certaines des formes extérieures sont sciemment reprises au rituel de la religion d'Ancien Régime ». Parmi ces formules, il y a évidemment celle de la « sainte guillotine » (p. 96).
} 
spectacle de la mort des aristocrates, ces «ennemis de la nation », elle admet ne plus pouvoir assister à aucune exécution depuis qu'elle a vu un vieillard, une jeune fille de quatorze ans, des religieuses, une jeune fille et sa mère menés à l'échafaud (p. 144). De manière manifeste, le texte bat en brèche une application des idéaux républicains qui a pour principal biais la violence. Ainsi, la prise de position nuancée d'un M. Ledru, l'un de convives dont les récits enchâssés composent Les Mille et Uns Fantômes, revêt une signification particulière. Tout en étant près des révolutionnaires (il est «lié » à Danton et à Camille Desmoulins), il œuvre à l'abolition de la peine de mort sur la bases d'arguments pathétiques (pensons à l'histoire de cet enfant noyé dans la mare de sang accumulé autour de la Place de la Révolution, p. 312) aussi bien que scientifiques, ses expériences ayant pour objectif de fournir des preuves sur lesquelles pourrait s'appuyer le discours abolitionniste (ibid.). Liées à l'introduction de plusieurs éléments fantastiques du récit, ses réflexions permettent de faire ressortir le caractère cruel de la peine de mort. Comme le montre l'exemple de Ledru, qui a vu sa bien-aimée lui adresser la parole après que sa tête eut été tranchée, les récits donnent la parole aux morts afin de vilipender les dérives révolutionnaires, de manière analogue à l'intervention du spectre d'Henri IV.

Il serait cependant erroné de voir dans cette prise de parole des morts une simple comparaison entre la peine de mort sous l'Ancien Régime et les exécutions pratiquées lors de la Terreur, comme le fait Anne-Marie Callet-Bianco lorsqu'elle écrit: « $[\mathrm{m}]$ ise à mort d'ancien régime contre exécutions révolutionnaires évoquées dans Solange: la Terreur se distingue par sa sanglante cruauté » (p. 34-35). Rappelons que la tête de Solange se met à parler alors que son Albert travaille 
afin de démontrer scientifiquement la cruauté de la peine de mort; les paroles de la morte constituent, ironiquement, la preuve que Ledru n'avait pu trouver au cours de ses longues soirées de recherches. Aussi un parallèle entre Solange, Jeanne et Arsène peut-il être établi : comme les têtes de Jeanne et Solange s'étaient animées pour dénoncer l'injustice qui avait causé leur mort, celle d'Arsène dénonce une iniquité, un meurtre qui avait certes ses motifs, mais qui est dépeint dans le roman comme atroce et blâmable. Ce que fait d'abord l'auteur ici, c'est insister sur la nature criminelle de l'acte de prendre la vie, qu'il soit perpétré de manière individuelle ou collective. Puis le texte révèle la contradiction flagrante qui voit les révolutionnaires reproduire les agissements qui ont motivé leur révolte au nom des valeurs républicaines. Enfin, il met en relief les dangers liés à une utilisation résolue, quoique ténébreuse, par le peuple de sa capacité d'action politique.

Voilà pourquoi, sous la plume de Dumas, cette pléthore de fantômes revêt une importance symbolique : ces spectres sont, à l'instar de son travail d'écriture, autant de représentations pathétiques des erreurs commises par le passé, autant de tentatives de ramener l'homme à la raison. Le contraste criant entre la promotion des libertés individuelles et les actions des révolutionnaires qui la bafouent, cette manière dont les hommes s'arrogent le droit de tuer au nom d'un idéal politique, permettent à l'écrivain de critiquer la peine de mort et, par ricochet, les artisans de la Terreur qui en ont fait un instrument «travaill[ant] à fonder une démocratie» (Arasse, p. 102). Le fantastique permet de rendre plus claire cette prise de position articulée par Ledru: la tête de Solange se met à parler et exprime ainsi la douleur des victimes injustement sacrifiées, tout comme l'avait fait la femme de Jacquemin. Est dénoncée, en 
somme, cette philosophie révolutionnaire que Hegel résume en ces termes : «[l]e particulier est trop petit en face de l'Universel: les individus sont donc sacrifiés et abandonnés. L'Idée paie le tribut de l'existence et de la caducité non par ellemême, mais au moyen des passions individuelles. » (voir Goulet, p. 72)

\section{Le «chavirement » du présent}

Dans l'Introduction des Mille et Un Fantômes, Alexandre Dumas insiste sur la nécessité d'atteindre l'équilibre fragile entre passé et avenir, c'est-à-dire la nécessité d'éviter le sacrifice du présent à un projet. Ce « chavirement » du présent ${ }^{5}$ acquiert un statut d'enjeu politique et social de premier ordre, à une époque où, ne serait-ce qu'en raison de l'instauration du suffrage universel masculin, le pouvoir dont le peuple devient le détenteur est désormais impossible à ignorer pour les penseurs et la classe politique. Alors que la victoire écrasante de la coalition du Parti de l'Ordre aux élections de 1848, puis l'accession de Louis Napoléon Bonaparte à la présidence et la remontée des forces conservatrices qui s'ensuivirent expriment pour Marx le refus populaire d'un régime politique au service de la bourgeoisie dont il renforce et consolide les privilèges aux dépens des classes inférieures ${ }^{6}-$, ces événements constituent, aux yeux de

\footnotetext{
5 « Le présent chavirerait sans cesse s'il n'était maintenu en équilibre par le poids de l'espérance et le contrepoids des souvenirs [...]» (p. 49).

6 Selon Marx, le geste des paysans reste néanmoins empreint d'une certaine maladresse : «C'est de ce jour seulement que date le Février des paysans français. Le symbole qui exprimait leur entrée dans le mouvement révolutionnaire, maladroit et rusé, coquin et naïf, lourdaud et sublime, superstition calculée, mascarade pathétique, anachronisme génial et stupide, espièglerie de l'histoire mondiale, - hiéroglyphe indéchiffrable pour la
} 
la bourgeoisie révolutionnaire et d'Alexandre Dumas, la preuve probante de l'incapacité du peuple à jouer adéquatement son rôle politique. Ils confirment le bien-fondé de la méfiance à l'égard de « la barbarie au sein de la civilisation » (Marx, Lutte des classes, p.125) qui avait incité les instigateurs de la Deuxième République à repousser les élections de 1848.

Il serait néanmoins malvenu de juger cette méfiance incompatible avec un attachement profond aux idéaux républicains. En critiquant ainsi la propension des mouvements et institutions politiques à recourir à la violence, Les Mille et Un Fantômes et La Femme au collier de velours établissent un net contraste entre l'importance du pouvoir sociopolitique du peuple, d'une part, et l'absence chez lui du discernement nécessaire à son exercice éclairé, d'autre part. Plus que la cause, ce sont ses défenseurs les plus ardents qui sont pris à partie. Ces récits sont imprégnés d'une méfiance à l'égard de la foule rassemblée autour d'un idéal politique qui la galvanise. L'ensemble de la représentation des sans-culottes servant la cause révolutionnaire - et dont la compréhension approximative des enjeux politiques et sociaux ne tempère aucunement le zèle, au contraire - l'indique indubitablement. Rien ne nous permet, par contre, de croire cette représentation sous-tendue par un quelconque projet de restauration de la monarchie. Plutôt qu'un légitimisme larvé, c'est un questionnement désabusé et tracassant sur le rôle social et politique $\mathrm{du}$ peuple qu'elle révèle, questionnement qui caractérise «les obsessions et le climat psychologique d'une génération» (p.36), celle d'Alexandre Dumas. Il s'agit d'un 
paradigme organisant la «lecture » du passé comme celle du présent (Certeau, p. 32). La préoccupation dumasienne vis-à-vis de l'action du peuple et des moyens de l'influencer (Dumas opte pour l'éducation) prélude en outre aux écrits de Gustave Le Bon $^{7}$. Elle a en outre partie liée avec l'esprit de la Constitution de 1848, qui incorpore les tiraillements entre l'attachement aux libertés et une crainte du désordre social et politique qui étaient le propre du républicanisme bourgeois de l'époque (Marx, 18 Brumaire, p. 29).

Car c'est une voie mitoyenne qui est promue par Dumas, en dépit $d u$ fait que l'ignorance du peuple s'est révélée rédhibitoire à l'atteinte d'un équilibre entre passé et avenir lors de la Révolution française, ce même équilibre que l'Introduction des Mille et Un Fantômes présentait comme un idéal politique. Si c'est au nom de la liberté que la garde nationale réprima le soulèvement des ouvriers en 1848, ce fut également en son nom que le passé fut violemment répudié en 1789. Voilà pourquoi, plus encore que la Terreur, c'est la réitération de ses erreurs les plus grossières au milieu du XIX ${ }^{\mathrm{e}}$ siècle qui est attaquée dans Les Mille et Un Fantômes, comme l'indique de manière peu équivoque l'Introduction. Ainsi, une telle représentation a beau nuancer le républicanisme dumasien, elle valorise en revanche les idéaux qui le sous-tendent. Pour Michel de Certeau, «[1]e discours historique explicite une identité sociale, non pas en tant qu'elle est "donnée" ou stable, mais en tant qu'elle se différencie d'une époque antérieure ou d'une autre société »

7 Ce dernier écrit dans l'introduction de Psychologie des foules: «La connaissance de la psychologie des foules constitue la ressource de l'homme d'État qui veut, non pas les gouverner - la chose est devenue aujourd'hui bien difficile - mais tout au moins ne pas être trop complètement gouverné par elles. » (p. 5) 
(p. 59). Le roman, chez Dumas, articulerait, en ce sens, une réflexion foncièrement historique. En effet, illustrer par l'absurde - comme il le fait dans Les Mille et Un Fantômes et La Femme au collier de velours - ce que sont la liberté et l'égalité, c'est préciser par le fait même ce qu'elles ne peuvent et ne devraient être, de manière à, ultimement, les consolider.

\section{Bibliographie}

ARASSE, Daniel. 1993 [1987], La Guillotine et l'imaginaire de la Terreur, Paris, Flammarion, coll. « Champs ».

ARROUS, Michel. 2003, «Introduction: Une lecture de l'Histoire », dans Michel Arrous (dir.), Dumas, une lecture de l'histoire, Paris, Maisonneuve \& Larose.

BERNARD, Claudie. 1996, Le Passé recomposé : le roman historique français du dix-neuvième siècle, Paris, Hachette, coll. « Hachette Université. Recherches littéraires ».

CERTEAU, Michel de. 1975, L'Écriture de l'histoire, Paris, Gallimard, coll. « Bibliothèque des histoires ».

DUMAS, Alexandre. 2006, Le Mois, [février 1848]. http://jad.ish - lyon.cnrs.fr/Numeros.php

—. 2006 [1849], Les Mille et Un Fantômes précédé de La Femme au collier de velours, Paris, Gallimard, coll. « Folio classique ».

GOUGH, Hugh. 1998, The Terror in the French Revolution, Houndmills, MacMillan Press, "Studies in European History » Series. 
GOULET, Jacques. 1983, Robespierre, la peine de mort et la terreur, Pantin, Le Castor Astral.

LE BON, Gustave. 1963 [1895], Psychologie des foules, Paris, Presses universitaires de France, coll. «Bibliothèque historique ».

LUKACS, Georges. 1965, Le Roman historique, Paris, Payot, coll. " Bibliothèque historique ».

MARX, Karl. 1976 [1891], Le 18 Brumaire de Louis Bonaparte, Paris, Éditions sociales, coll. « Classiques du Marxisme »;

- 1984 [1850], Les Luttes de classes en France: 1848-1850, Paris, Messidor/Éditions sociales, coll. « Essentiel».

MOMBERT, Sarah. 2003, " "Apprendre l'Histoire au peuple": Alexandre Dumas vulgarisateur », dans Michel Arrous (dir.), Dumas, une lecture de l'histoire, Paris, Maisonneuve \& Larose. TROYAT, Henri. 2005. Alexandre Dumas: le cinquième mousquetaire, Paris, Grasset.

\section{Résumé}

Le portrait pathétique de la Terreur brossé par Alexandre Dumas dans La Femme au collier de velours et Les Milles et Un Fantômes porte un jugement sévère sur le recours à la violence des révolutionnaires. L'utilisation effrénée de la peine de mort y apparaît comme une manifestation de l'immaturité d'une classe exerçant maladroitement un pouvoir politique nouvellement acquis. Mais si cette représentation de l'Histoire est teintée d'une certaine appréhension du pouvoir populaire, c'est moins les valeurs de liberté, d'égalité et de démocratie que leur galvaudage qui est critiqué dans ces récits. À cet égard, 
l'interprétation du passé se révèle, chez Dumas, inspirée du présent et résolument tournée vers l'avenir.

\begin{abstract}
The pathetic portrait of the Reign of Terror built up by Alexandre Dumas in La Femme au collier de velours and Les Milles et Un Fantômes censures the insurgents' use of violence. In these texts, the frantic use of the death penalty emphasizes the callowness of a class that clumsily handles its newly acquired political powers. Yet, if this representation of History is somewhat tainted by a fear of the power resting in the people's hands, it is not the values of liberty, equality, and democracy, but their degradation that it criticizes. In this respect, Dumas' reflection on the past is inspired by the present, and is purposefully oriented toward the future.
\end{abstract}

